Topological Wiener-Wintner ergodic theorems via non-abelian Lie group extensions

Santos, Sara I. and Walkden, Charles

2007

MIMS EPrint: 2007.190

Manchester Institute for Mathematical Sciences

School of Mathematics

The University of Manchester

\footnotetext{
Reports available from: http://eprints.maths.manchester.ac.uk/

And by contacting: The MIMS Secretary

School of Mathematics

The University of Manchester

Manchester, M13 9PL, UK
} 


\title{
Topological Wiener-Wintner ergodic theorems via non-abelian Lie group extensions
}

\author{
SARA I. SANTOS and CHARLES WALKDEN \\ School of Mathematics, The University of Manchester, Oxford Road, Manchester \\ M13 9PL, UK \\ (e-mail: cwalkden@maths.man.ac.uk)
}

(Received 3 May 2006 and accepted in revised form 9 October 2006)

Abstract. We generalize a series of topological Wiener-Wintner ergodic theorems due to Walters to the context of group extensions of measure-preserving transformations where the group is a non-abelian compact Lie group. Applications to random ergodic theorems for a shift map are given.

\section{Introduction}

Let $T: X \rightarrow X$ be an ergodic transformation of a probability space $(X, \mathcal{B}, \mu)$ and let $f: X \rightarrow \mathbb{C}$ be an $L^{1}$ function. Let $K=\{z \in \mathbb{C}|| z \mid=1\}$ denote the unit circle in $\mathbb{C}$. The classical Wiener-Wintner ergodic theorem is concerned with the almost sure (a.s.) convergence of

$$
\frac{1}{n} \sum_{j=0}^{n-1} z^{-j} f\left(T^{j} x\right)
$$

as $n \rightarrow \infty$, where $z \in K$. When $z=1$ this is Birkhoff's ergodic theorem and (1) converges almost surely to $\int f d \mu$. By considering the direct product $T_{z}: X \times K \rightarrow X \times K$ given by $T_{z}(x, y)=(T(x), y z)$ one can easily deduce the almost sure convergence of (1) from Birkhoff's ergodic theorem. However, it is not clear that the set of full measure on which convergence holds can be chosen to be independent of $z$; this is the content of the WienerWintner ergodic theorem.

Let $U: L^{2}(X, \mathcal{B}, \mu) \rightarrow L^{2}(X, \mathcal{B}, \mu)$ be the unitary operator defined by $U f=f \circ T$. The convergence of (1) is dependent on whether $z$ is an eigenvalue for $U$. For each $z \in K$ define $P_{z}: L^{2}(X, \mathcal{B}, \mu) \rightarrow L^{2}(X, \mathcal{B}, \mu)$ by setting $P_{z} f$ to be the projection of $f$ onto the eigenspace for $U$ corresponding to the eigenvalue $z$ (when $z$ is an eigenvalue of $U$ ), and $P_{z} f=0$ otherwise. Then the Wiener-Wintner ergodic theorem can be stated as follows. 
THEOREM 1.1. (Wiener-Wintner ergodic theorem [WW]) Let $f \in L^{2}(X, \mathcal{B}, \mu)$. Then there exists a set $X_{f}$ with $\mu\left(X_{f}\right)=1$ such that for all $x \in X_{f}$ and all $z \in K$,

$$
\frac{1}{n} \sum_{j=0}^{n-1} z^{-j} f\left(T^{j} x\right) \rightarrow P_{z} f(x)
$$

as $n \rightarrow \infty$.

(The restriction that $f \in L^{2}(X, \mathcal{B}, \mu)$ can be weakened to $f \in L^{1}(X, \mathcal{B}, \mu)$ (see [WW]); in this case, there exists a set $X_{f}$ of full measure for which the right-hand side of (2) converges for all $x \in X_{f}$ and all $z \in K$.)

Topological analogues of Theorem 1.1 in the vein of Oxtoby's ergodic theorem are known $[\mathbf{R}, \mathbf{A}]$; here $T$ is a uniquely ergodic continuous transformation of a compact metric space, $f$ is continuous, and uniform convergence (in both $x$ and $z$ ) of (2) is required.

Generalizations of these results are proved in [Wa]. Here, the convergence of quantities of the form

$$
\frac{1}{n} \sum_{j=0}^{n-1} \phi(x) \phi(T x) \cdots \phi\left(T^{j-1} x\right) f\left(T^{j} x\right),
$$

where $T$ is a continuous transformation of a compact metric space $X$ and $\phi: X \rightarrow K$ is a continuous function, is studied. The convergence depends on the ergodic properties of the circle skew-product

$$
T_{\phi}: X \times K \rightarrow X \times K:(x, y) \mapsto(T x, y \phi(x)) .
$$

As a particular case, the following is proved in [Wa].

THEOREM 1.2. (Walters' topological Wiener-Wintner theorem) Suppose that $T_{\phi}: X \times$ $K \rightarrow X \times K$ is ergodic with respect to the invariant measure $\mu \times \lambda$ where $\lambda$ denotes Lebesgue measure on $K$. Then there exists a set $X(\mu)$ with $\mu(X(\mu))=1$ such that for any continuous function $f: X \rightarrow \mathbb{C}$ and any $x \in X(\mu)$

$$
\lim _{n \rightarrow \infty} \frac{1}{n} \sum_{j=0}^{n-1} \phi(x) \phi(T x) \cdots \phi\left(T^{j-1} x\right) f\left(T^{j} x\right)=0 .
$$

In this paper we generalize the results of [Wa] to the case of an arbitrary compact Lie group $G$. Recall that a compact Lie group has a faithful representation in $O(d)$, the group of real $d \times d$ orthogonal matrices, and so there is no loss in generality in assuming that $G<O(d)$ is a closed subgroup.

Let $T$ be a continuous transformation of a compact metric space $X$, let $\phi: X \rightarrow O(d)$ and let $f: X \rightarrow \mathbb{R}^{d}$ be continuous. We are then interested in the convergence of

$$
\frac{1}{n} \sum_{j=0}^{n-1} \phi(x) \phi(T x) \cdots \phi\left(T^{j-1} x\right) f\left(T^{j} x\right)
$$

as $n \rightarrow \infty$.

Let

$$
\operatorname{Fix}(G)=\left\{v \in \mathbb{R}^{d} \mid g v=v \text { for all } g \in G\right\}
$$


denote the subspace of vectors in $\mathbb{R}^{d}$ that are fixed under all elements of $G$. Let $\operatorname{proj}_{\text {Fix }(G)}: \mathbb{R}^{d} \rightarrow \operatorname{Fix}(G) \subset \mathbb{R}^{d}$ denote the orthogonal projection onto this subspace.

Define the skew-product

$$
T_{\phi}: X \times G \rightarrow X \times G:(x, y) \mapsto(T x, y \phi(x)) .
$$

It is easily seen that if $\lambda_{G}$ denotes Haar measure on $G$ then the measure $\mu \times \lambda_{G}$ is invariant under $T_{\phi}$. The ergodic properties of this measure will form a key part of our analysis. As a particular case, we prove the following result.

THEOREM 1.3. Suppose that $T$ is a continuous transformation of a compact metric space $X$ and that $\mu$ is an ergodic Borel probability measure. Suppose that $T_{\phi}$ is ergodic with respect to $\mu \times \lambda_{G}$. Then there exists a set $X(\mu)$ with $\mu(X(\mu))=1$ such that for any continuous function $f: X \rightarrow \mathbb{R}^{d}$ and any $x \in X(\mu)$

$$
\frac{1}{n} \sum_{j=0}^{n-1} \phi(x) \phi(T x) \cdots \phi\left(T^{j-1} x\right) f\left(T^{j} x\right) \rightarrow \operatorname{proj}_{\text {Fix }(G)} \int f d \mu
$$

as $n \rightarrow \infty$.

A compact Lie group $G$ also has a faithful representation in $U(d)$, the group of $d \times d$ unitary matrices acting on $\mathbb{C}^{d}$. All of our results can also be stated in this setting with only notational alterations.

\section{The measurable structure of skew-products}

The ergodic decomposition for skew-products is well known [KN1, KN2]. Here we summarize the facts that we shall need.

If $Y$ is a compact metric space then we denote by $M(Y)$ the space of Borel probability measures on $Y$ equipped with the weak* topology. If $T: Y \rightarrow Y$ is a continuous transformation, then $M(Y, T) \subset M(Y)$ denotes the non-empty subset of $T$-invariant measures and $E(Y, T) \subset M(Y, T)$ denotes the non-empty subset of ergodic measures.

Let $\pi: X \times G \rightarrow X:(x, y) \mapsto x$ denote the natural projection. A measure $v$ on $X \times G$ is said to project to a measure $\mu$ on $X$ if $\nu\left(\pi^{-1}(B)\right)=\mu(B)$ for all Borel subsets $B$ of $X$. We will sometimes say that $v$ is a lift of $\mu$. Throughout we shall denote by $\lambda_{G}$ the Haar measure on $G$; if there is no risk of ambiguity then we shall simply write $\lambda$. The measure $\mu \times \lambda$ is sometimes called the Haar lift of $\mu$.

There is a natural left $G$-action on $X \times G$ defined by $l_{g}(x, y)=(x, g y)$. This $G$-action commutes with the skew-product $T_{\phi}$ defined in (3). Let $\Sigma^{d}=\left\{z \in \mathbb{C}^{d} \mid\|z\|=1\right\}$, where $\|\cdot\|$ denotes the inner-product norm on $\mathbb{C}^{d}$. We denote by $\mathcal{R}(G)$ the (equivalence classes of) continuous irreducible unitary representations of $G$. Let $\gamma$ be a continuous $d$-dimensional irreducible unitary representation of $G$. We say that a measurable function $W: X \times G \rightarrow \Sigma^{d}$ is a $\gamma$-function if $W\left(l_{g}(x, y)\right)=\gamma(g) W(x, y)$ for all $(x, y) \in G$ and all $g \in G$.

The following provides well-known criteria for the ergodicity of $\mu \times \lambda_{G}$.

Proposition 2.1. [KN1, KN2] Let $(X, \mathcal{B}, \mu)$ be a probability space, let $T$ be a measurable transformation of $X$ and let $\mu$ be an ergodic measure. Let $G$ be a 
compact Lie group. For a measurable function $\phi: X \rightarrow G$ define the skew-product $T_{\phi}$ as in (3). Then the following are equivalent:

(i) $T_{\phi}$ is ergodic with respect to $\mu \times \lambda_{G}$;

(ii) $\mu \times \lambda_{G}$ is the only $T_{\phi}$-invariant measure on $X \times G$ that projects to $\mu$;

(iii) if $W_{\gamma}$ is a measurable $\gamma$-function such that $W_{\gamma} \circ T_{\phi}=W_{\gamma} \mu \times \lambda_{G}$-a.e. (almost everywhere) for some continuous $d$-dimensional irreducible unitary representation $\gamma$ of $G$, then $d=1, \gamma$ is the trivial representation, and $W_{\gamma}$ is constant $\mu \times \lambda_{G}$-a.e.;

(iv) if $w: X \rightarrow \mathbb{C}^{d}$ is a measurable function such that

$$
w(T x)=\gamma(\phi(x)) w(x) \mu-a . e .
$$

for some continuous $d$-dimensional irreducible unitary representation $\gamma$ of $G$, then $d=1, \gamma$ is the trivial representation, and $w$ is constant a.e.

Remark. As $\gamma$ is a unitary representation, if (4) holds then $|w|$ is a $T$-invariant function. By ergodicity, $|w|$ is constant. Hence there is no loss in generality in assuming that $w$ takes values in $\Sigma^{d}$.

In the case when the Haar lift of $\mu$ to $G$ is not ergodic, the following result describes the ergodic decomposition of $\mu \times \lambda_{G}$.

PROPOSITION 2.2. Suppose that $T_{\phi}$ is not ergodic with respect to $\mu \times \lambda_{G}$. Then there is a proper Lie subgroup $H$ of $G$ and a measurable map $s: X \rightarrow G$ such that for each $g \in G$ the sets

$$
X_{g, s}=\{(x, g H s(x)) \in X \times G \mid x \in X\}
$$

are $T_{\phi}$-invariant. Furthermore, $T_{\phi}: X_{g, s} \rightarrow X_{g, s}$ is ergodic with respect to the probability measure $\hat{\mu}_{g, s}$ defined by

$$
\hat{\mu}_{g, s}=S^{*}\left(\mu \times l_{g}^{*} \lambda_{H}\right),
$$

where $S: X \times G \rightarrow X \times G$ is defined by $S(x, y)=(x, y s(x))$. Moreover, $\hat{\mu}_{g, s}$ projects to $\mu$ and is the unique $T_{\phi}$-invariant probability measure on $X_{g, s}$ with this property.

As this will be a key fact in our analysis, we indicate the proof and refer to [KN2] for details.

Proof (sketch). Let $v \in E\left(X \times G, T_{\phi}\right)$ be ergodic and suppose that $v$ projects to $\mu$. For each irreducible representation class $[\gamma] \in \mathcal{R}(G)$ of dimension $d_{\gamma}$ suppose that there are precisely $l_{\gamma} T_{\phi}$-invariant $\gamma$-functions $W_{\gamma}^{i}, 1 \leq i \leq l_{\gamma}$ (possibly $l_{\gamma}=0$ for some $\gamma \in$ $\mathcal{R}(G)$ ). Since $v$ is ergodic we have that $W_{\gamma}^{i}=a_{\gamma}^{i} v$-a.e., for $1 \leq i \leq l_{\gamma}$ for some constant vectors $a_{\gamma}^{i}$.

Suppose that $v \neq \mu \times \lambda$. Let

$$
\begin{gathered}
H=\left\{g \in G \mid \gamma(g) a_{\gamma}^{i}=a_{\gamma}^{i}, 1 \leq i \leq l_{\gamma},[\gamma] \in \mathcal{R}(G)\right\}, \\
E=\left\{x \in X \mid W_{\gamma}^{i}(x, y)=a_{\gamma}^{i}, 1 \leq i \leq l_{\gamma},[\gamma] \in \mathcal{R}(G)\right\} .
\end{gathered}
$$

Then $H$ is a closed, hence Lie, subgroup of $G$ and is a proper subgroup as we are assuming that $\mu \times \lambda$ is not ergodic. The set $E$ is $T_{\phi}$-invariant and satisfies $v(E)=1$; moreover, the set $E^{\prime}=\pi(E) \subset X$ satisfies $\mu\left(E^{\prime}\right)=1$. 
For $x \in E^{\prime}$ we choose $s(x)$ in a measurable way such that $(x, s(x)) \in E$. As $E$ is $T_{\phi}$-invariant, we have that $(T(x), s(x) \phi(x)) \in E$ and $(T(x), s(T(x))) \in E$. From the definitions of $H$ and $E$ it is easy to see that if $(x, y) \in E$ and $g(x, y) \in E$ then $g \in H$. Hence $s(x) \phi(x) s(T x)^{-1} \in H$.

Define $\phi^{\prime}(x): X \rightarrow H$ by $s(x) \phi(x) s(T x)^{-1}$ if $x \in E^{\prime}$ and arbitrarily otherwise. Then the map $S(x, y)=(x, y s(x))$ conjugates $T_{\phi^{\prime}}$ to $T_{\phi}$. Moreover, for each $g \in G, T_{\phi^{\prime}}$ leaves the sets $l_{g}(X \times H)=X \times g H$ invariant and one can check using [KN2] that $T_{\phi^{\prime}}$ is ergodic on $X \times g H$ with respect to $l_{g}^{*}\left(\mu \times \lambda_{H}\right)$ (and so by Proposition 2.1(ii) is the only ergodic measure for $T_{\phi^{\prime}}$ on $g(X \times H)$ that projects to $\left.\mu\right)$. Hence for each $g \in G, T_{\phi}$ leaves the sets

$$
X_{g, s}=\{(x, g H s(x)) \mid x \in X\}
$$

invariant and is ergodic with respect to the measure $\hat{\mu}_{g, s}$ as in the statement of the proposition.

Remark. Choquet theory $[\mathbf{P h}]$ and the above results allow us to describe the set of $T_{\phi}$-invariant probability measures that project to $\mu$. If $v$ is a $T_{\phi}$-invariant probability measure then there exists a Borel probability measure $\alpha$ supported on $G / H$ such that (with a small abuse of notation)

$$
v=\int_{G / H} S^{*}\left(\mu \times l_{g}^{*} \lambda_{H}\right) d \alpha(g H) .
$$

Remark. The subgroup $H$ can be characterized as

$$
H=\bigcap_{\gamma \in \mathcal{R}(G)}\left\{\operatorname{Ann}(\gamma) \mid \text { there exists a measurable } T_{\phi} \text {-invariant } \gamma \text {-function }\right\}
$$

where $\operatorname{Ann}(\gamma)=\{g \in G \mid \gamma(g)=e\}$.

Remark. In the case where $T$ is an Anosov diffeomorphism or an Axiom $A$ diffeomorphism restricted to a locally maximal hyperbolic set and $\phi: X \rightarrow G$ is Hölder, then a topological decomposition of the skew-product is possible [B, PP2]. In particular, one can take $s(x)$ in Proposition 2.2 to be Hölder.

\section{Integration and invariant measures for the skew-product}

Here and throughout, if $X$ and $Y$ are metric spaces then we denote by $C(X, Y)$ the space of continuous functions from $X$ to $Y$.

Let $G$ be a compact Lie group. Then $G$ has a faithful representation in $O(d)$ for some $d \geq 1$ and so there is no loss in assuming that $G<O(d)$ is a closed subgroup.

Let $f \in C\left(X, \mathbb{R}^{d}\right)$ and define $F: X \times G \rightarrow \mathbb{R}^{d}$ by $F(x, g)=g f(x)$. We are interested in integrating this function with respect to a $T_{\phi}$-invariant measure. Let

$$
\operatorname{proj}_{\text {Fix }(G)}(\cdot): \mathbb{R}^{d} \rightarrow \operatorname{Fix}(G)
$$

denote the orthogonal projection from $\mathbb{R}^{d}$ onto the subspace $\operatorname{Fix}(G)=\left\{v \in \mathbb{R}^{d} \mid g v=v\right.$ for all $g \in G\}$.

Proposition 3.1. With $F$ defined as above we have

$$
\int F d \mu \times \lambda_{G}=\operatorname{proj}_{\mathrm{Fix}(G)} \int f d \mu .
$$


Proof. We need to prove that

$$
\int_{G} g d \lambda_{G}(g)=\operatorname{proj}_{\mathrm{Fix}(G)}(\cdot)
$$

Recall that $G$ is a subgroup of $O(d)$, which in turn can be viewed as a subgroup of the group $U(d)$ of $d \times d$ unitary matrices. Thus we can see the inclusion $\iota: G \rightarrow U(d), \iota(g)=$ $\left[g_{i, j}\right]_{1 \leq i, j \leq d}$, as a $d$-dimensional unitary representation of $G$. Thus we can decompose $\iota$ as a sum of irreducible unitary representations $\iota_{1}, \ldots, \iota_{k}$. Hence we can write

$$
\iota(g)=\left[g_{i, j}\right]=\left(\begin{array}{cccc}
\iota_{1}(g) & 0 & \cdots & 0 \\
0 & \iota_{2}(g) & \cdots & 0 \\
\vdots & & \ddots & \\
0 & \ldots & 0 & \iota_{k}(g)
\end{array}\right)
$$

in an appropriate basis. Thus each entry of $\int_{G} g d \lambda_{G}(g)$ is the integral of either 0 or $\left(\iota_{\ell}\right)_{i, j}$ with respect to Haar measure on $G$. But $\int_{G}\left(\iota_{\ell}\right)_{i, j} d \lambda_{G}=0$ unless $\iota_{\ell}$ is equivalent to the trivial representation, by the Peter-Weyl theorem. Therefore the only non-zero entries of the matrix $\int_{G} g d \lambda_{G}$ are those that correspond to invariant one-dimensional subspaces, i.e. to $\operatorname{Fix}(G)$. Thus

$$
\int F(x, g) d \mu \times \lambda_{G}=\int_{G} g d \lambda_{G} \int_{X} f d \mu=\operatorname{proj}_{\mathrm{Fix}(G)} \int_{X} f d \mu .
$$

Remarks. (i) If $\operatorname{Fix}(G)=\{0\}$ then the integral in (7) is equal to 0 for all $f \in C\left(X, \mathbb{R}^{d}\right)$. This is the case, for example, if $G=S O(d)$.

(ii) An element of $S O$ (3) can be represented as a rotation through some angle about an axis in $\mathbb{R}^{3}$. If $G$ is a subgroup of $S O(3)$ that contains two elements with distinct axes then $\operatorname{Fix}(G)=\{0\}$.

Let $T: X \rightarrow X$ be a continuous transformation of a compact metric space and let $\mu \in E(X, T)$. Let $X(\mu)$ denote the set of generic points for $T$ :

$$
X(\mu)=\left\{x \in X \mid \frac{1}{n} \sum_{j=0}^{n-1} f\left(T^{j} x\right) \rightarrow \int f d \mu \text { as } n \rightarrow \infty \text { for all continuous } f: X \rightarrow \mathbb{R}\right\} .
$$

By the compactness of $X$ and Birkhoff's ergodic theorem, we know that $\mu(X(\mu))=1$. The following is an adaptation of a result in [Wa] to our setting. Here and throughout $\delta_{z}$ denotes the Dirac delta-measure supported on the point $z$.

PROPOSITION 3.2. Let $T$ be a continuous transformation of a compact metric space $X$ and let $\mu \in E(X, T)$.

(i) Let $x \in X(\mu)$ and $g \in G$. Then every weak* limit point of

$$
\frac{1}{n} \sum_{j=0}^{n-1} \delta_{T_{\phi}^{j}(x, g)}
$$

in $M(X \times G)$ is a $T_{\phi}$-invariant probability measure that projects to $\mu$. 
(ii) Let $\phi_{r}: X \rightarrow G$ be a sequence of continuous functions that converges uniformly to $\phi, x_{r}$ be a sequence of points in $X, n_{r}$ a strictly increasing sequence of integers, and $g \in G$. Then any weak* limit point of

$$
\frac{1}{n_{r}} \sum_{j=0}^{n_{r}-1} \delta_{T_{\phi_{r}}^{j}\left(x_{r}, g\right)}
$$

as $r \rightarrow \infty$ is a $T_{\phi}$-invariant probability measure.

(iii) If, in addition to the hypotheses in (ii), we assume that $x_{r} \in X(\mu)$ then any weak* limit point of

$$
\frac{1}{n_{r}} \sum_{j=0}^{n_{r}-1} \delta_{T_{\phi_{r}}^{j}\left(x_{r}, g\right)}
$$

projects to $\mu$.

Proof. Statements (i) and (iii) follow easily from (ii). We prove (ii), adapting the argument from $[\mathbf{W a}$. Let $\rightarrow$ denote weak $*$ convergence.

Suppose that

$$
\frac{1}{n_{r_{k}}} \sum_{j=0}^{n_{r_{k}}-1} \delta_{T_{\phi_{r_{k}}}}^{j}\left(x_{r_{k}}, g\right) \rightarrow v
$$

as $k \rightarrow \infty$. Let $H: X \times O(d) \rightarrow \mathbb{R}^{d}$ be a continuous map of the form $H(x, g)=$ $\left(h_{1}(x) \ell_{1}(g), \ldots, h_{n}(x) \ell_{n}(g)\right)$ where the $\ell_{i}: O(d) \rightarrow \mathbb{R}$ are Lipschitz continuous. As such functions are uniformly dense in the space of continuous functions $X \times O(d) \rightarrow \mathbb{R}$ (by the Stone-Weierstrass theorem), it suffices to prove that $\int H \circ T_{\phi} d v=\int H d v$.

Now

$$
\begin{aligned}
\int H \circ T_{\phi} d v-\int H d v \\
=\left[\begin{array}{c}
\left.\int H_{1} \circ T_{\phi} d v-\int H_{1} d v\right] \\
\vdots \\
\left.\int H_{n} \circ T_{\phi} d v-\int H_{n} d v\right] \\
= \\
\left.\lim _{k \rightarrow \infty} \frac{1}{n_{r_{k}}} \sum_{j=0}^{n_{r_{k}}-1}\left|H_{1}\left(T_{\phi} \circ T_{\phi_{r_{k}}}^{j}\left(x_{r_{k}}, g\right)\right)-H_{1}\left(T_{\phi_{r_{k}}}^{j}\left(x_{r_{k}}, g\right)\right)\right|\right] \\
\left.\lim _{k \rightarrow \infty} \frac{1}{n_{r_{k}}} \sum_{j=0}^{n_{r_{k}}-1}\left|H_{n}\left(T_{\phi} \circ T_{\phi_{r_{k}}}^{j}\left(x_{r_{k}}, g\right)\right)-H_{n}\left(T_{\phi_{r_{k}}}^{j}\left(x_{r_{k}}, g\right)\right)\right|\right]
\end{array}\right] .
\end{aligned}
$$


The $i$ th entry in this vector is equal to

$$
\begin{aligned}
\lim _{k \rightarrow \infty} & \frac{1}{n_{r_{k}}} \sum_{j=0}^{n_{r_{k}}-1}\left|H_{i}\left(T_{\phi} \circ T_{\phi_{r_{k}}}^{j}\left(x_{r_{k}}, g\right)\right)-H_{i}\left(T_{\phi_{r_{k}}}^{j}\left(x_{r_{k}}, g\right)\right)\right| \\
= & \lim _{k \rightarrow \infty}\left(\sum_{j=0}^{n_{r_{k}}-1}\left(H_{i}\left(T_{\phi} \circ T_{\phi_{r_{k}}}^{j}\left(x_{r_{k}}, g\right)\right)-H_{i}\left(T_{\phi_{r_{k}}}^{j}\left(x_{r_{k}}, g\right)\right)\right)\right. \\
& \left.+H_{i}\left(T_{\phi_{r_{k}}}^{n_{r_{k}}}\left(x_{r_{k}}, g\right)\right)-H_{i}\left(x_{r_{k}}, g\right)\right) \\
= & \lim _{k \rightarrow \infty}\left(\sum _ { j = 0 } ^ { n _ { r _ { k } } - 1 } H _ { i } ( T ^ { j + 1 } x _ { r _ { k } } ) \left[\ell_{k}\left(g \sum_{l=0}^{j-1} \phi_{r_{k}}\left(T^{l} x_{r_{k}}\right) \phi\left(T^{j} x_{r_{k}}\right)\right)\right.\right. \\
& \left.\left.-\ell_{k}\left(g \sum_{l=0}^{j-1} \phi_{r_{k}}\left(T^{l} x_{r_{k}}\right) \phi_{r_{k}}\left(T^{j} x_{r_{k}}\right)\right)\right]\right) .
\end{aligned}
$$

Hence $\int H \circ T_{\phi} d v-\int H d v$ is bounded above in norm by

$$
\max _{i}\left|\ell_{i}\right|_{\text {Lip }}\|h\|_{\infty}\left\|\phi-\phi_{r_{k}}\right\|
$$

(where $|\cdot|_{\text {Lip }}$ denotes the Lipschitz constant), which converges to 0 as $k \rightarrow \infty$.

\section{Topological Wiener-Wintner theorems}

We use the results of $\S \S 2$ and 3 to deduce a series of Wiener-Wintner ergodic theorems, of which Theorem 1.3 is a special case. These results generalize those in [Wa]. In particular, the results in [Wa] follow from the special case of $G=S O(2)$ acting on $\mathbb{R}^{2}$.

Throughout, $G$ denotes a closed subgroup of $O(d)$.

THEOREM 4.1. Let $T: X \rightarrow X$ be a continuous transformation of a compact metric $X$, let $\mu \in E(X, T)$ and let $X(\mu)$ denote the set of $\mu$-generic points. Let $\phi \in C(X, G)$.

(i) Suppose that $T_{\phi}: X \times G \rightarrow X \times G$ is ergodic with respect to $\mu \times \lambda_{G}$. Then for all $f \in C\left(X, \mathbb{R}^{d}\right)$ and all $x \in X(\mu)$ we have

$$
\lim _{n \rightarrow \infty} \frac{1}{n} \sum_{j=0}^{n-1} \phi(x) \phi(T x) \cdots \phi\left(T^{j-1} x\right) f\left(T^{j} x\right)=\operatorname{proj}_{\text {Fix }(G)} \int f d \mu .
$$

(ii) Suppose that $T_{\phi}: X \times G \rightarrow X \times G$ is not ergodic with respect to $\mu \times \lambda_{G}$. Let $H$ be as in (6). Then there exists a measurable function $s: X \rightarrow G$ with $s(x) \phi(x) s(T x)^{-1} \in H$ such that for all $f \in C\left(X, \mathbb{R}^{d}\right)$ and all $x \in X(\mu)$ we have

$$
\lim _{n \rightarrow \infty} \frac{1}{n} \sum_{j=0}^{n-1} \phi(x) \phi(T x) \cdots \phi\left(T^{j-1} x\right) f\left(T^{j} x\right)=s(x)^{-1} \operatorname{proj}_{\text {Fix }(H)} \int s f d \mu .
$$

Proof. We prove (i). Suppose that the claimed convergence fails. Then there exists $x \in X(\mu), \varepsilon>0$ and an increasing sequence of integers $n_{k}$ such that

$$
\left|\frac{1}{n_{k}} \sum_{j=0}^{n_{k}-1} \phi(x) \phi(T x) \cdots \phi\left(T^{j-1} x\right) f\left(T^{j} x\right)-\operatorname{proj}_{\mathrm{Fix}(G)} \int f d \mu\right| \geq \varepsilon .
$$


Note that iterating (3) yields that

$$
T_{\phi}^{n}(x, y)=\left(T^{n} x, y \phi(x) \phi(T x) \cdots \phi\left(T^{n-1} x\right)\right) .
$$

Define $F: X \times G \rightarrow \mathbb{R}^{d}$ by $F(x, g)=g f(x)$. Then (8) can be rewritten as

$$
\left|\int F d v_{k}-\operatorname{proj}_{\mathrm{Fix}(G)} \int f d \mu\right| \geq \varepsilon
$$

where $F(x, g)=g f(x)$ and

$$
v_{k}=\frac{1}{n_{k}} \sum_{j=0}^{n_{k}-1} \delta_{T_{\phi}^{j}(x, e)} .
$$

By Proposition 3.2(i) it follows that $v_{k}$ has a subsequence that weak* converges to a $T_{\phi}$-invariant measure $v$ that projects to $\mu$. Assuming that $\mu \times \lambda_{G}$ is ergodic, it follows from Proposition 2.1(ii) that $v=\mu \times \lambda$. By Proposition 3.1 we have that $\int F d v=$ $\operatorname{proj}_{\mathrm{Fix}(G)} \int f d \mu$, a contradiction.

The proof of (ii) is similar. If the claimed convergence fails, then there exists $x \in X(\mu)$, $\varepsilon>0$ and an increasing sequence of integers $n_{k}$ such that

$$
\left|\frac{1}{n_{k}} \sum_{j=0}^{n_{k}-1} \phi(x) \phi(T x) \cdots \phi\left(T^{j-1} x\right) f\left(T^{j} x\right)-s(x)^{-1} \operatorname{proj}_{\operatorname{Fix}(H)} \int s f d \mu\right| \geq \varepsilon .
$$

This can be rewritten as

$$
\left|\int F d v_{k}-s(x)^{-1} \operatorname{proj}_{\text {Fix }(H)} \int s f d \mu\right| \geq \varepsilon,
$$

where $F(x, g)=g f(x)$ and $v_{k}$ is as in (9).

As $T_{\phi}$ is not ergodic with respect to $\mu \times \lambda_{G}$, we can use Proposition 2.1 to find a closed subgroup $H$ and a measurable function $s: X \rightarrow G$ such that the sets $X_{g, s}$ are invariant and support a unique $T_{\phi}$-invariant measure $\hat{\mu}_{g, s}$ that projects to $\mu$.

Note that $(x, e) \in X_{g, s}$ where $g=s(x)^{-1}$. Hence $v_{k}$ is supported on $X_{g, s}$. By compactness, $v_{k}$ has a convergent subsequence that weak $*$ converges to a $T_{\phi}$-invariant measure supported on $X_{g, s}$. By Proposition 3.2(i) this weak* limit must be equal to $\hat{\mu}_{g, s}$. Now

$$
\begin{aligned}
\int F d \hat{\mu}_{g, s} & =\int F d S^{*}\left(\mu \times l_{g}^{*} \lambda_{H}\right) \\
& =\int F(x, g h s(x)) d \mu \times \lambda_{H} \\
& =\int g h s(x) f(x) d \mu \times \lambda_{H} \\
& =g \int_{H} h d \lambda_{H} \int s f d \mu \\
& =s(x)^{-1} \operatorname{proj}_{\operatorname{Fix}(H)} \int s f d \mu
\end{aligned}
$$

a contradiction. 
COROLLARY 4.2. Let $T$ be a continuous transformation of a compact metric space and let $\mu \in E(X, T)$. Let $\phi \in C(X, G)$. Then the following are equivalent:

(i) there does not exist a measurable function $l: X \rightarrow \Sigma^{d}$ such that $l(x)=\phi(x) l(T x)$ for $\mu$-a.e. $x$;

(ii) for all continuous functions $f: X \rightarrow \mathbb{R}^{d}$ and for all $x \in X(\mu)$,

$$
\lim _{n \rightarrow \infty} \frac{1}{n} \sum_{j=0}^{n-1} \phi(x) \cdots \phi\left(T^{j-1} x\right) f\left(T^{j} x\right)=0
$$

Proof. We prove that (i) implies (ii). By Theorem 4.1 we know that the limit in (10) exists for all $x \in X(\mu)$; denote this limit by $l_{\phi, f}(x)$. As $X(\mu)$ is $T_{\phi}$-invariant, we have that

$$
\frac{1}{n} \sum_{j=0}^{n-1} \phi(T x) \cdots \phi\left(T^{j-1}(T x)\right) f\left(T^{j}(T x)\right) \rightarrow l_{\phi, f}(T x) .
$$

Observing that

$$
\sum_{j=0}^{n-1} \phi(T x) \cdots \phi\left(T^{j-1}(T x)\right) f\left(T^{j}(T x)\right)=\sum_{j=0}^{n} \phi(x) \cdots \phi\left(T^{j-1} x\right) f\left(T^{j} x\right)-f(x)
$$

it follows that $l_{\phi, f}(x)=\phi(x) l_{\phi, f}(T x)$. As $\left|l_{\phi, f}\right|=\left|l_{\phi, f}\right| \circ T \mu$-a.e., it follows that $\left|l_{\phi, f}\right|$ is constant $\mu$-a.e. If $\left|l_{\phi, f}\right| \neq 0 \mu$-a.e. then by replacing $f$ by $f /\left|l_{\phi, f}\right|$ we obtain a measurable function $l_{\phi, f}: X \rightarrow \Sigma^{d}$. Hence if (i) holds then the limit in (10) must be zero for all $x \in X(\mu)$.

Conversely, suppose that there exists a measurable function $l: X \rightarrow \Sigma^{d}$ such that $l(x)=\phi(x) l(T x)$ for all $x \in X(\mu)$. Iterating this identity we see that $\phi\left(T^{j} x\right)^{-1} \cdots \phi(x)^{-1} l(x)=l\left(T^{j} x\right)$ for $x \in X(\mu)$. Then, letting $\langle\cdot, \cdot\rangle$ denote the Euclidean inner product in $\mathbb{R}^{d}$, we have that for all continuous $f: X \rightarrow \mathbb{R}^{d}$ and a.e. $x$

$$
\begin{aligned}
\langle l(x) & \left., \frac{1}{n} \sum_{j=0}^{n-1} \phi(x) \cdots \phi\left(T^{j-1} x\right) f\left(T^{j} x\right)\right\rangle \\
= & \frac{1}{n} \sum_{j=0}^{n-1}\left\langle\phi\left(T^{j} x\right)^{-1} \cdots \phi(x)^{-1} l(x), f\left(T^{j} x\right)\right\rangle \\
= & \frac{1}{n} \sum_{j=0}^{n-1}\left\langle l\left(T^{j} x\right), f\left(T^{j} x\right)\right\rangle \\
& \rightarrow \int\langle l, f\rangle d \mu
\end{aligned}
$$

as $n \rightarrow \infty$ by Birkhoff's ergodic theorem. As $f$ is arbitrary, it follows that $l$ must be zero $\mu$-a.e.

We can use Proposition 3.2(iii) in place of Proposition 3.2(i) in the proof of Theorem 4.1 together with Corollary 4.2 to obtain the following result which gives a uniform limit over a compact subset of functions $\phi \in C(X, G)$. 
PROPOSITION 4.3. Let $T$ be a continuous transformation of a compact metric space and let $\mu \in E(X, T)$. Let $\mathcal{K} \subset C(X, G)$ be a uniformly compact subset of functions. Suppose that for each $\phi \in \mathcal{K}$ there is no measurable function $l: X \rightarrow \Sigma^{d}$ such that $l(x)=\phi(x) l(T x) \mu$-a.e. Then for each $f \in C\left(X, \mathbb{R}^{d}\right)$

$$
\lim _{n \rightarrow \infty} \frac{1}{n} \sum_{j=0}^{n-1} \phi(x) \cdots \phi\left(T^{j-1} x\right) f\left(T^{j} x\right)=0
$$

where the convergence is uniform in $\phi \in \mathcal{K}$.

Proof. By Theorem 4.1 we know that for each $\phi \in \mathcal{K}$

$$
\lim _{n \rightarrow \infty} \frac{1}{n} \sum_{j=0}^{n-1} \phi(x) \cdots \phi\left(T^{j-1} x\right) f\left(T^{j} x\right)
$$

converges for $x \in X(\mu)$. By Corollary 4.2, the limit is 0 . It remains to check that the convergence is uniform. Suppose not. Then there exists $x \in X(\mu)$ and $\varepsilon>0$ such that for all $k \geq 1$ there exists $n_{k} \geq k$ and $\phi_{k} \in \mathcal{K}$ such that

$$
\left|\frac{1}{n_{k}} \sum_{j=0}^{n_{k}-1} \phi_{k}(x) \cdots \phi_{k}\left(T^{j-1} x\right) f\left(T^{j} x\right)\right| \geq \varepsilon .
$$

Writing $F(x, g)=g f(x)$ and

$$
v_{k}=\frac{1}{n_{k}} \sum_{j=0}^{n_{k}-1} \delta_{T_{\phi_{k}}^{j}(x, e)}
$$

the above can be rewritten as

$$
\left|\int F d v_{k}\right| \geq \varepsilon
$$

Choose a subsequence $\phi_{k_{i}}$ of the $\phi_{k}$ that converges uniformly to $\phi \in C(X, G)$. By compactness and Proposition 3.2(iii), $v_{k_{i}}$ has a convergent subsequence that weak* converges to a $T_{\phi}$-invariant probability measure $v$ that projects to $\mu$.

If $T_{\phi}$ is ergodic with respect to $\mu \times \lambda_{G}$ then $\nu=\mu$ and $\int F d \mu \times \lambda_{G}=0$, a contradiction.

Suppose that $T_{\phi}$ is not ergodic with respect to $\mu \times \lambda_{G}$. Then by the remarks following Proposition 2.2, there exists a closed subgroup $H$ of $G$, a measurable function $s: X \rightarrow G$ and a Borel probability measure $\alpha$ supported on $G / H$ such that

$$
v=\int_{G / H} S^{*}\left(\mu \times l_{g}^{*} \lambda_{H}\right) d \alpha
$$

where $S(x, y)=(x, y s(x))$. Then

$$
\int F d v=\int_{G / H} g H d \alpha \operatorname{proj}_{\mathrm{Fix}(H)} \int s f d \mu .
$$

We claim that $\int s f d \mu=0$. To see this, note that by Theorem 4.1

$$
\lim _{n \rightarrow \infty} \frac{1}{n} \sum_{j=0}^{n-1} \phi(x) \cdots \phi\left(T^{j-1} x\right) f\left(T^{j} x\right)=s(x)^{-1} \operatorname{proj}_{\mathrm{Fix}(H)} \int s f d \mu ;
$$


hence by Corollary 4.2

$$
s(x)^{-1} \operatorname{proj}_{\text {Fix }(H)} \int s f d \mu=0
$$

for $x \in X(\mu)$. As $s(x)$ is orthogonal, it follows that $\operatorname{proj}_{\text {Fix }(H)} \int s f d \mu=0$. Hence $\int F d v=0$, a contradiction.

Recall that a continuous transformation of a compact metric space is said to be uniquely ergodic if there is a unique invariant probability measure. If we assume that $T$ is uniquely ergodic then we can obtain an analogue of Proposition 4.3 where the convergence holds uniformly for all $x \in X$ and $\phi$ in a compact subset of $C(X, G)$.

COROLlARY 4.4. Let $T: X \rightarrow X$ be a uniquely ergodic continuous transformation of a compact metric space with unique invariant probability measure $\mu$. Let $\mathcal{K} \subset C(X, G)$ be a uniformly compact collection of functions. Suppose that for each $\phi \in \mathcal{K}$ there is no measurable function $l: X \rightarrow \Sigma^{d}$ such that $l(x)=\phi(x) l(T x) \mu$-a.e. Then, for each $f \in C\left(X, \mathbb{R}^{d}\right)$,

$$
\lim _{n \rightarrow \infty} \frac{1}{n} \sum_{j=0}^{n-1} \phi(x) \cdots \phi\left(T^{j-1}\right) f\left(T^{j} x\right)=0,
$$

where the convergence is uniform in $(x, \phi) \in X \times \mathcal{K}$.

Proof. By Oxtoby's ergodic theorem [O], if $T$ is uniquely ergodic then we can take $X(\mu)=X$. Hence by Theorem 4.1 and Corollary 4.2 we have that

$$
\lim _{n \rightarrow \infty} \frac{1}{n} \sum_{j=0}^{n-1} \phi(x) \cdots \phi\left(T^{j-1} x\right) f\left(T^{j} x\right)=0 .
$$

Suppose that this convergence is not uniform in $(x, \phi) \in X \times \mathcal{K}$. Then there exists $\varepsilon>0$ such that for all $k \in \mathbb{N}$ there exists $n_{k} \geq k, x_{k} \in X$ and $\phi_{k} \in \mathcal{K}$ such that

$$
\left|\frac{1}{n_{k}} \sum_{j=0}^{n_{k}-1} \phi_{k}\left(x_{k}\right) \cdots \phi_{k}\left(T^{j-1} x_{k}\right) f\left(T^{j} x_{k}\right)\right| \geq \varepsilon .
$$

Letting $F(x, g)=g f(x)$, this can be rewritten as

$$
\left|\int F d v_{k}\right| \geq \varepsilon
$$

where

$$
v_{k}=\frac{1}{n_{k}} \sum_{j=0}^{n_{k}-1} \delta_{T_{\phi_{k}}^{j}\left(x_{k}, e\right)} .
$$

Choose a subsequence $\phi_{k_{i}}$ such that $\phi_{k_{i}} \rightarrow \phi$. Choose a subsequence of $v_{k_{i}}$ that weak* converges to $v$. By Proposition 3.2, $v$ is a $T_{\phi}$-invariant probability measure. As $v$ projects to a $T$-invariant measure and $T$ is uniquely ergodic, it follows that $v$ projects to $\mu$. The proof now proceeds as in the proof of Proposition 4.3. 
Finally, we give a measurable version of Theorem 4.1.

Proposition 4.5. Suppose that $(X, \mathcal{B}, \mu)$ is a Lebesgue probability space, $T: X \rightarrow X$ is a measure-preserving transformation, and $\phi: X \rightarrow G$ is a measurable function. Let $f \in L^{1}\left(X ; \mathbb{R}^{d}\right)$. Then

$$
\frac{1}{n} \sum_{j=0}^{n-1} \phi(x) \cdots \phi\left(T^{j-1} x\right) f\left(T^{j} x\right) \rightarrow \hat{f}(x) \mu-a . e .,
$$

where $\hat{f} \in L^{1}\left(X, \mathbb{R}^{d}\right)$ is such that $\hat{f}(x)=\phi(x) \hat{f}(T x) \mu$-a.e.

Proof. By $[\mathbf{F u}]$, there exists a compact metric space $X^{\prime}$, a continuous transformation $T^{\prime}: X^{\prime} \rightarrow X^{\prime}$, a measure $\mu^{\prime}$ on the completion $\overline{\mathcal{B}}(X)$ of the Borel $\sigma$-algebra of $X^{\prime}$, and a measure-preserving bimeasurable isomorphism $V:(X, \mathcal{B}, \mu) \rightarrow\left(X^{\prime}, \overline{\mathcal{B}}(X), \mu^{\prime}\right)$ between $T$ and $T^{\prime}$. Moreover, $\phi \circ V^{-1}: X^{\prime} \rightarrow G$ is equal $\mu^{\prime}$-a.e. to a continuous function. Hence, up to measurable isomorphism, there is no loss in assuming that $T$ is a continuous transformation of a compact metric space, $\mu$ is a probability measure on the completion of the Borel $\sigma$-algebra, and $\phi$ is continuous.

First suppose that $\mu$ is ergodic. If $f \in C\left(X, \mathbb{R}^{d}\right)$ then it follows from Theorem 4.1 and the remarks made in the proof of Corollary 4.2 that there exists $X_{f} \in \mathcal{B}$ with $\mu\left(X_{f}\right)=1$ such that

$$
\frac{1}{n} \sum_{j=0}^{n-1} \phi(x) \cdots \phi\left(T^{j-1} x\right) f\left(T^{j} x\right) \rightarrow \hat{f}(x) \text { for } x \in X_{f},
$$

where $\hat{f}$ is a measurable function such that $\hat{f}(x)=\phi(x) \hat{f}(T x)$ a.e.

Suppose that $f \in L^{1}\left(X, \mathbb{R}^{d}\right)$. Let $\varepsilon>0$. Choose $f_{k} \in C\left(X, \mathbb{R}^{d}\right)$ such that $\int\left|f-f_{k}\right| d \mu<1 / k$. By Birkhoff's ergodic theorem, there exists $X_{(k)}$ with $\mu\left(X_{(k)}\right)=1$ such that

$$
\frac{1}{n} \sum_{j=0}^{n-1}\left|f-f_{k}\right|\left(T^{j} x\right) d \mu \rightarrow \int\left|f-f_{k}\right| d \mu<\frac{1}{k} \quad \text { for } x \in X_{(k)}
$$

as $n \rightarrow \infty$. Denoting $(1 / n) \sum_{j=0}^{n-1} \phi(x) \cdots \phi\left(T^{j-1} x\right) f\left(T^{j} x\right)$ by $S_{n, \phi} f(x)$ we have that

$$
\begin{aligned}
\left|S_{n, \phi} f(x)-S_{m, \phi} f(x)\right| \leq & \left|S_{n, \phi} f(x)-S_{n, \phi} f_{k}(x)\right|+\left|S_{n, \phi} f_{k}(x)-S_{m, \phi} f_{k}(x)\right| \\
& +\left|S_{m, \phi} f_{k}(x)-S_{m, \phi} f(x)\right| .
\end{aligned}
$$

Now

$$
\begin{aligned}
\left|S_{n} f(x)-S_{n} f_{k}(x)\right| & =\left|\frac{1}{n} \sum_{j=0}^{n-1} \phi(x) \cdots \phi\left(T^{j-1} x\right)\left(f\left(T^{j} x\right)-f_{k}\left(T^{j} x\right)\right)\right| \\
& \leq \frac{1}{n} \sum_{j=0}^{n-1}\left|f-f_{k}\right|\left(T^{j} x\right)
\end{aligned}
$$

as $\phi(\cdot)$ is orthogonal. 
If $n, m$ are sufficiently large, then $\left|S_{n, \phi} f(x)-S_{n, \phi} f_{k}(x)\right| \leq \varepsilon$ by Theorem 4.1. Let $x \in \bigcap_{k=1}^{\infty} X_{f_{k}} \cap \bigcap_{k=1}^{\infty} X_{(k)}$, a set of full $\mu$-measure. Choose $k$ such that $1 / k<\varepsilon$. Then if $n, m$ are sufficiently large,

$$
\left|S_{n, \phi} f(x)-S_{m, \phi} f(x)\right| \leq \varepsilon,
$$

so that $S_{n, \phi}(x)$ is Cauchy and therefore converges.

Now suppose that $\mu$ is not ergodic. Then the set

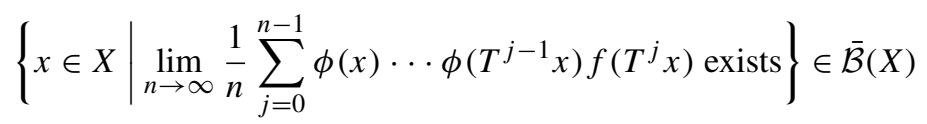

has full measure for all measures in the ergodic decomposition of $\mu$, and so has full $\mu$-measure.

Finally, observe that as $\phi(\cdot)$ is orthogonal,

$$
\begin{aligned}
|\hat{f}(x)| & \leq \lim _{n \rightarrow \infty} \frac{1}{n} \sum_{j=0}^{n-1}\left\|\phi(x) \cdots \phi\left(T^{j-1} x\right)\right\|\left|f\left(T^{j} x\right)\right| \\
& =\lim _{n \rightarrow \infty} \frac{1}{n} \sum_{j=0}^{n-1}\left|f\left(T^{j} x\right)\right|,
\end{aligned}
$$

which is an integrable function by Birkhoff's ergodic theorem.

\section{Applications to shifts}

The results of $\S 4$ in the case where the base is a shift map can be used to deduce several random ergodic theorems. These are related to results in [Wa], where such theorems are proved in the case of commuting unitary operators acting on a Hilbert space; in the result below the unitary operators do not need to commute, but the Hilbert space on which they act is required to be finite-dimensional.

Let $\Sigma_{k}=\left\{\left(x_{j}\right)_{j=0}^{\infty} \mid x_{j} \in\{1, \ldots, k\}\right\}$ denote the full two-sided $k$-shift with shift map $\sigma: \Sigma_{k} \rightarrow \Sigma_{k}$ defined by $(\sigma(x))_{j}=x_{j+1}$.

For $x, y \in \Sigma_{k}, x \neq y$, we define $n(x, y)=\sup \left\{n \mid x_{j}=y_{j}\right.$ for $\left.|j| \leq n\right\}$. Then for each $\theta \in(0,1)$ we define a metric $d_{\theta}$ by setting $d_{\theta}(x, y)=\theta^{n(x, y)}$ if $x \neq y$ and $d_{\theta}(x, y)=0$ otherwise. We let $F_{\theta}\left(\Sigma_{k}, \mathbb{R}\right)$ denote the space of real-valued functions that are Lipschitz continuous with respect to $d_{\theta}$; by an abuse of notation if $f \in F_{\theta}\left(\Sigma_{k}, \mathbb{R}\right)$ for some $\theta \in(0,1)$ then we say that $f$ is Hölder continuous. Let $\psi \in F_{\theta}\left(\Sigma_{k}, \mathbb{R}\right)$. Then there is a unique measure $\mu_{\psi} \in M\left(\Sigma_{k}, \sigma\right)$ such that

$$
h\left(\mu_{\psi}\right)+\int \psi d \mu_{\psi}=\sup _{\mu \in M\left(\Sigma_{k}, \sigma\right)} h(\mu)+\int \psi d \mu,
$$

where $h(\cdot)$ denotes measure-theoretic entropy [PP1]. We call $\mu_{\psi}$ the equilibrium state corresponding to the potential $\psi$. Equilibrium states corresponding to Hölder continuous potentials are ergodic.

We shall need the following result, which is surely known, but we were unable to locate a reference. 
LEMMA 5.1. Let $G$ be a compact group and let $S$ be a closed semigroup of $G$. Then $S$ is a group.

Proof. We prove that if $x \in S, x \neq e$, then $x^{-1} \in S$. Let $S_{x}=\operatorname{cl}\left\{x^{n} \mid n \in \mathbb{N}\right\}$ denote the closed semigroup generated by $x$. Note that $S_{x}$ is abelian.

Consider the sequence $x^{n} \in S, n \geq 0$. By compactness, we may find a convergent subsequence $x^{n_{k}}$ such that $x^{n_{k}}$ converges to a limit $x^{\infty} \in S_{x}$ as $n_{k} \rightarrow \infty$. By taking a further subsequence, we may assume that $2 n_{k}<n_{k+1}$. Let $m_{k}=n_{k+1}-n_{k}-1 \geq n_{k}$. Then $x^{m_{k}} x x^{n_{k}}=x^{n_{k+1}}$. By taking further subsequences, if necessary, we can assume that the sequence $m_{k}$ is increasing and that $x^{m_{k}}$ converges to a limit $y \in S_{x}$. Hence $y x x^{\infty}=x^{\infty}$, so that $x^{-1}=y \in S$.

Proposition 5.2. Let $k \geq d$. Let $\sigma: \Sigma_{k} \rightarrow \Sigma_{k}$ be the full-shift on $k$ symbols and let $\mu \in E\left(\Sigma_{k}, \sigma\right)$ be an equilibrium state corresponding to a Hölder continuous potential. Then there exists an open dense subset $\Delta \subset S O(d)^{k}$ such that if $\left(A_{1}, \ldots, A_{k}\right) \in \Delta$, $f \in C\left(\Sigma_{k}, \mathbb{R}^{d}\right)$ and $x \in \Sigma_{k}(\mu)$ then

$$
\lim _{n \rightarrow \infty} \frac{1}{n} \sum_{j=0}^{n-1} A_{x_{0}} \cdots A_{x_{j-1}} f\left(\sigma^{j} x\right)=0 .
$$

Proof. By [Fi] there exists an open dense set $\Delta \subset S O(d)^{k}$ such that each $k$-tuple $A=$ $\left(A_{1}, \ldots, A_{k}\right) \in \Delta$ topologically generates $S O(d)$.

Choose such a $k$-tuple $A$ and form the skew-product $\sigma_{A}: \Sigma_{k} \times S O(d) \rightarrow \Sigma_{k} \times$ $S O(d):(x, y) \mapsto\left(\sigma(x), y A_{x_{0}}\right)$. We claim that $\sigma_{A}$ is ergodic with respect to $\mu \times \lambda_{S O}(d)$. By Proposition 2.1(iii) this is equivalent to showing that there are only trivial solutions to

$$
w(\sigma(x))=\gamma\left(A_{x_{0}}\right) w(x) \quad \mu \text {-a.e. }
$$

where $w: \Sigma_{k} \rightarrow \Sigma^{d}$ is measurable and $\gamma$ is a continuous irreducible unitary representation of $S O(d)$. By [PP2] the existence of measurable solutions to (11) is equivalent to the existence of continuous solutions. By [P], continuous solutions to (11) depend on just one coordinate of $x$ which we may take to be $x_{0}$. Hence (11) can be written as $w\left(x_{1}\right)=\gamma\left(A_{x_{0}}\right) w\left(x_{0}\right)$. By Lemma 5.1 we see that $w(i)=\gamma(g) w_{i}$ for all $g \in S O(d)$, $1 \leq i \leq k$. Since $\gamma$ is irreducible, it follows that $\gamma$ is the trivial representation and $w$ is constant. Hence $\sigma_{A}$ is ergodic.

Noting that $\operatorname{Fix}(S O(d))=\mathbb{R}^{d}$, the result follows from Theorem 4.1.

If we take $f(x)=\chi_{E}(x) v$, where $v \in \mathbb{R}^{d}$ is a fixed vector and $E$ is a finite union of cylinders, in Theorem 4.1, we obtain the following corollary. This is related to a result in [Wa] for commuting unitary operators acting on a Hilbert space; in our setting the operators need not commute, but they must act on a finite-dimensional Hilbert space. Note that if $\phi(x)=A_{x_{0}}$ then, by (6), $H$ is the closed subgroup generated by $\left\{A_{1}, \ldots, A_{k}\right\}$. 
Corollary 5.3. Let $\sigma$ and $\mu$ be as in Proposition 5.2. Let $A=\left(A_{1}, \ldots, A_{k}\right) \in$ $S O(d)^{k}$. Let $E \subset \Sigma_{k}$ be a finite union of cylinders. Then for all $x \in \Sigma_{k}(\mu)$ and $v \in \mathbb{C}^{d}$,

$$
\lim _{n \rightarrow \infty} \frac{1}{n} \sum_{j=0}^{n-1} \chi_{E}(\sigma(x)) A_{x_{0}} \cdots A_{x_{j-1}} v=\mu(E) \operatorname{proj}_{\text {Fix }(\langle A\rangle)} v,
$$

where $\langle A\rangle$ denotes the closed subgroup generated by $A_{1}, \ldots, A_{k}$.

In [Wa], it is proved that

$$
\frac{1}{n} \sum_{j=0}^{n-1} z_{x_{0}} \cdots z_{x_{j-1}}\left(f\left(\sigma^{j} x\right)-\int f d \mu\right) \rightarrow 0
$$

uniformly in $\left(z_{1}, \ldots, z_{k}\right) \in K^{k}$, where $f \in C\left(\Sigma_{k}, \mathbb{C}\right)$ and $\mu$ is an equilibrium state corresponding to a Hölder continuous potential. The following result generalizes this from $K$ to $S O(d)$.

Proposition 5.4. Let $k \geq d$. Let $\sigma: \Sigma_{k} \rightarrow \Sigma_{k}$ be the full-shift on $k$ symbols and let $\mu \in E\left(\Sigma_{k}, \sigma\right)$ be an equilibrium state corresponding to a Hölder continuous potential. Let $f \in C\left(\Sigma_{k}, \mathbb{R}^{d}\right)$. Then for all $x \in \Sigma_{k}(\mu)$ we have

$$
\lim _{n \rightarrow \infty} \frac{1}{n} \sum_{j=0}^{n-1} A_{x_{0}} \cdots A_{x_{j-1}}\left(f\left(\sigma^{j} x\right)-\int f d \mu\right)=0
$$

uniformly for $\left(A_{1}, \ldots, A_{k}\right) \in S O(d)^{k}$.

Proof. Let $A=\left(A_{1}, \ldots, A_{k}\right) \in S O(d)^{k}$ and form the skew-product $\sigma_{A}$ as above.

It is sufficient to prove the result in the case when $\int f d \mu=0$. By Proposition 4.5 we know that

$$
\frac{1}{n} \sum_{j=0}^{n-1} A_{x_{0}} \cdots A_{x_{j-1}} f\left(\sigma^{j} x\right) \rightarrow \hat{f}(x)
$$

where $\hat{f}$ is a measurable function such that

$$
\hat{f}(x)=A_{x_{0}} \hat{f}(\sigma(x))
$$

for $x \in \Sigma_{k}(\mu)$. We claim that $\hat{f}=0$.

If $\sigma_{A}$ is ergodic with respect to $\mu \times \lambda_{S O(d)}$ then

$$
\hat{f}(x)=\operatorname{proj}_{\mathrm{Fix}(S O(d))} \int f d \mu=0 .
$$

Suppose that $\sigma_{A}$ is not ergodic with respect to $\mu \times \lambda_{S O(d)}$. Then Theorem 4.1 implies that

$$
\hat{f}(x)=s(x)^{-1} \operatorname{proj}_{\mathrm{Fix}(H)} \int s f d \mu
$$

for some closed subgroup $H<S O(d)$ and measurable $s: \Sigma_{k} \rightarrow S O(d)$. By [PP2], any measurable solution $\hat{f}$ to (13) has a continuous version; by $[\mathbf{P}]$, any continuous solution to (13) depends on just one coordinate. Hence $s(x)$ depends on one coordinate, which we 
may assume to be $x_{0}$. (We remark that this also follows from a close inspection of the construction of $s$ in the proof of Proposition 2.2.)

It follows from (13) and (14) that

$$
s(i)^{-1} \operatorname{proj}_{\text {Fix }(H)} \int s f d \mu=A_{i} s(j)^{-1} \operatorname{proj}_{\text {Fix }(H)} \int s f d \mu .
$$

As $f$ is arbitrary, this implies that $s(i)^{-1}=A_{i} s(j)^{-1}$ on $\operatorname{Fix}(H)$ for all $1 \leq i, j \leq k$.

Let $l=\operatorname{dim} \operatorname{Fix}(H)$. By choosing a suitable basis, we can write $\mathbb{R}^{d}=\operatorname{Fix}(H) \oplus$ Fix $(H)^{\perp}$. With respect to this basis, we can write

$$
s(i)=\left[\begin{array}{cc}
S_{i} & T_{i} \\
U_{i} & V_{i}
\end{array}\right], \quad A_{i}=\left[\begin{array}{cc}
I & 0 \\
0 & B_{i}
\end{array}\right],
$$

where $S_{i}$ is an $l \times l$ matrix, $I$ is the $l \times l$ identity matrix, $V_{i}$ and $B_{i}$ are $(n-l) \times(n-l)$ matrices, and $T_{i}$ is an $l \times(n-l)$ matrix. Then $s(i)^{-1}=A_{i} s(j)^{-1}$ implies that $S_{i}=S_{j}$ and $T_{i} B_{i}=T_{j}$. From this it is easy to see that

$$
\operatorname{proj}_{\text {Fix }(H)} s_{j}=\operatorname{proj}_{\text {Fix }(H)} s_{i} A_{i}
$$

for any $1 \leq i, j \leq k$.

Let $[j]=\left\{x \in \Sigma_{k} \mid x_{0}=j\right\}$ and fix any $i_{0} \in\{1, \ldots, k\}$. Then

$$
\begin{aligned}
\operatorname{proj}_{\text {Fix }(H)} \int s f d \mu & =\operatorname{proj}_{\text {Fix }(H)} \sum_{j=1}^{k} \int_{[j]} s(j) f d \mu \\
& =\operatorname{proj}_{\text {Fix }(H)} s\left(i_{0}\right) A_{i_{0}} \sum_{j=1}^{k} \int_{[j]} f d \mu \\
& =\operatorname{proj}_{\text {Fix }(H)} s\left(i_{0}\right) A_{i_{0}} \int f d \mu \\
& =0 .
\end{aligned}
$$

Hence for any $k$-tuple of matrices $A=\left(A_{1}, \ldots, A_{k}\right) \in S O(d)^{k}$, the limit $\hat{f}$ in (12) is equal to 0 . The same argument as in the proof of Proposition 4.3 can then be used to show that this convergence is uniform in $A$.

Acknowledgements. We would like to thank Mike Field and Mick McCrudden for useful comments. The first author was financially supported by Fundação para a Ciência e Tecnologia, Portugal, grant SFRH/BD/6921/2001.

\section{REFERENCES}

[A] I. Assani. Wiener-Wintner Ergodic Theorems. World Scientific Publishing, River Edge, NJ, 2003.

[B] M. I. Brin. The topology of group extensions of $C$-systems. Math. Notes 18 (1975), 453-465.

[Fi] M. Field. Generating sets for compact semisimple Lie groups. Proc. Amer. Math. Soc. 127 (1999), 3361-3365.

[Fu] H. Furstenberg. Disjointness in ergodic theory, minimal sets, and a problem in Diophantine approximation. Math. Systems Theory 1 (1967), 1-49. 
[KN1] H. B. Keynes and D. Newton. The structure of ergodic measures for compact group extensions. Israel J. Math. 18 (1974), 363-389.

[KN2] H. B. Keynes and D. Newton. Ergodic measures for non-abelian compact group extensions. Israel J. Math. 18 (1974), 363-389.

[O] J. C. Oxtoby. Ergodic sets. Bull. Amer. Math. Soc. 58 (1952), 116-136.

[P] W. Parry. A cocycle equation for shifts. Symbolic Dynamics and its Applications (Contemporary Mathematics, 135). Ed. P. Walters. American Mathematical Society, Providence, RI, 1992, pp. 327-333.

[PP1] W. Parry and M. Pollicott. Zeta functions and the periodic orbit structure of hyperbolic dynamics. Astérisque 187-188 (1990).

[PP2] W. Parry and M. Pollicott. The Livsic cocycle equation for compact Lie group extensions of hyperbolic systems. J. London Math. Soc. 56 (1997), 405-416.

[Ph] R. Phelps. Lectures on Choquet's Theorem. Van Nostrand, New York, 1966.

[R] E. A. Robinson. On uniform convergence in the Wiener-Wintner theorem. J. London Math. Soc. 49 (1994), 493-501.

[Wa] P. Walters. Topological Wiener-Wintner ergodic theorems and a random $L^{2}$ ergodic theorem. Ergod. Th. \& Dynam. Sys. 16 (1996), 179-206.

[WW] N. Wiener and A. Wintner. Harmonic analysis and ergodic theory. Amer. J. Math. 63 (1941), 415-426. 